UDC 629.7.05

O.A. Sushchenko, Cand. Sci. (Eng.)

A.V. Plotnikov

\title{
MOVING OBJECT ATTITUDE DETERMINATION BY MEANS OF REDUNDANT NON-ORTHOGONAL SYSTEMS OF SENSORS
}

NAU Institute of Electronics and Control Systems, e-mail: fsu@nau.edu.ua

\begin{abstract}
Peculiarities of moving object attitude determination by means of redundant non-orthogonal systems of sensors are considered. Advantages of this way of providing reliability are shown. Possible cases of sensor orientation in redundant non-orthogonal systems are studied. Their comparative analysis is carried out.
\end{abstract}

\section{Introduction}

Functional and engineering complication of modern transportation facilities makes it necessary to create new promising systems for moving object control. One of the most important tasks of moving object control is to determine spatial attitude of an object with respect to a given reference frame. Then it is necessary to provide movement of the object along a given trajectory according to a specified program. In other words, it is necessary to solve problems of attitude and navigation. One of the basic ways to solve these problems is the use of inertial reference systems. These systems have a number of advantages as they are self-contained, noiseproof, universal and provide a wide range of navigational measurements. Operation of inertial reference systems is based on integration of accelerations measured on a moving object by means of accelerometers whose spatial attitude is determined by gyro devices.

According to design, inertial reference systems are divided into systems with gyro-stabilized platform and strap-down systems [1]. In systems of the first type, sensors of kinematic parameters are mounted on a gyro-stabilized platform located in the gimbals. In this case, the platform realizes a reference frame on a moving object. The attitude of the object is determined with respect to this reference frame. In strap-down systems sensors of kinematic parameters are rigidly fixed on a moving object. Navigation and attitude parameters are determined analytically by means of a board computer. The high accuracy within a limited operating period and simplicity of navigational information processing algorithms are advantages of systems of the first type. However, systems of the second type are believed to be especially promising nowadays. Such systems are small in mass and size owing to the absence of a gyro-stabilized platform and their associated servo systems. They are well adapted to the increase of navigational information reliability owing to the use of appropriate hardware and software tools. These systems have great possibilities for increasing navigational information accuracy by algorithmic methods.
According to modern trends in designing attitude and navigation systems, inertial reference systems with a gyro-stabilized platform are created only if either high accuracy or cost reduction based on the previous experience is required [2]. One of the lines in navigational systems development is designing devices capable of determining the full spatial attitude of a moving object, the functionalities of such devices being close to those of inertial reference systems but requiring significantly smaller cost for their designing. They include directional and vertical gyros for determining angles of yaw, pitch, roll and accelerometers used for integral correction of a vertical gyro. Such correction makes a vertical gyro non-susceptible to disturbances caused by external accelerations. Such measuring instruments as accelerometers provide information about the speed and trajectory of a moving object after appropriate integration. So, it is possible to refer such devices to inertial reference systems [2]. Among the main characteristics of navigation and attitude systems are accuracy, reliability and cost. During the last years, navigational systems are characterized by a significant decrease of their mass and size as well as by a great drop in cost. It is connected with the use of miniature sensors of kinematic parameters. Significant progress in computer engineering is also of great importance for essentially extending the functionalities of on-board computers and giving possibilities for upgrading navigational systems a software tools.

The modern sensors of kinematic parameters are quite reliable, but when developing a system we must take into account such an effective instrument of increasing reliability as redundancy, which would, inevitably, increase the mass and size of the system. But miniaturization of kinematic parameter sensors allows us to view redundancy as a possible way of increasing the system's reliability. It is known that there are ways to reserve not sensors of kinematic parameters only but measuring reference frames too. In the first case, a traditional three-dimensional orthogonal reference frame is used and sensors are mounted along each axis. 
The redundancy is provided by means of some sensors mounted along each axis. Surely, the use of more than two sensors is not reasonable in this case. Under such an approach a failure of two sensors may lead to a failure of the whole navigation system.

The likelihood of a situation like this is little, although the latter is possible. Redundancy of measuring reference frames is based on mounting sensors along the axes of a certain geometrical figure. It provides redundancy of projections of kinematic parameters along the axes of the basic reference frame connected with a moving object. The redundancy of measuring reference frames provides more information about kinematic parameter projections onto the axes of the basic reference frame at a smaller mass and size [3].

\section{Analysis of the latest publications}

Basic approaches to the redundancy of measuring reference frames are presented in $[3 ; 4]$. But now that significant progress has been achieved in the miniaturization of sensors, the development and analysis of the considered approach requires special research. It should be noted that the problem of sensor redundancy must be solved providing a given accuracy and cost limitations.

It is also necessary to take into account the great progress in the field of computing techniques. Primarily, this progress is connected with a significant increase of information processing speed, which allows us to develop sophisticated algorithms for sensor serviceability analysis and to create a new configuration retaining the serviceability of the whole navigation system in case of a failure of any sensor. We may say that the use of these methods essentially improves the reliability of a moving object's spatial attitude determination.

\section{The goal of the study}

The goal of this paper is to investigate the peculiarities of using non-orthogonal redundant systems of sensors for the determination of the spatial attitude of a moving object as well as to estimate its influence on the performance characteristics of navigational systems.

\section{Analysis of different ways of orientation of non-orthogonal redundant systems of sensors}

Modern inertial reference systems use floated gyros, tuned rotor gyros, accelerometers, angular rate sensors, angular acceleration sensors as sensors of kinematic parameters.

The way of providing redundancy, which is considered here, is suitable for single-axis sensors, such as accelerometers, angular rate sensors, angular acceleration sensors.
It is known that there are two ways of building redundant measuring reference frames [3, 4]:

- the use of a cone as a figure of symmetry;

- the arrangement of sensor sensitivity axes relative to the faces of regular polyhedrons;

In its turn, when using a cone as a figure of symmetry, there are the following ways of building non-orthogonal redundant systems:

- all the sensors are oriented along the cone's generators;

- one of the sensors is lied along the axis of symmetry of the cone and all the rest - along the cone's generators through equal angles $\alpha=2 \pi / n$;

- multicone arrangement with an axis of symmetry common for all cones;

- multicone arrangement with axes of symmetry individual for each cone.

Examples of using regular polyhedrons for creation of measuring redundant reference frames are presented in fig. 1, 2 .

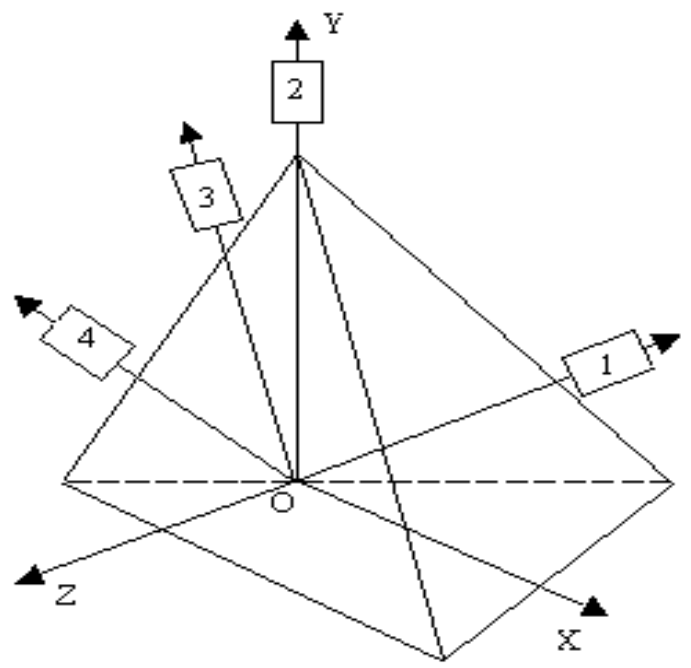

$a$

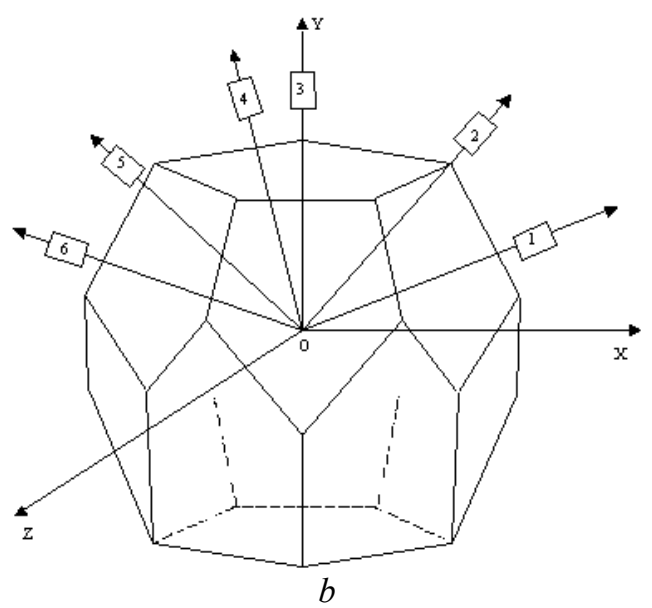

Fig. 1. The use of a tetrahedron (a) and a dodecahedron (b) for creation of a measuring redundant reference frame:

1-6-sensors 
In the first case, the orientation of sensors relative to the faces of a tetrahedron is shown. In the second case, the orientation of sensors relative to the faces of a dodecahedron is presented. Four and six sensors respectively are used here.Examples of using the orientation of sensors along the cone's generators only and along the cone's generators and axis of symmetry are presented in fig. 2 .
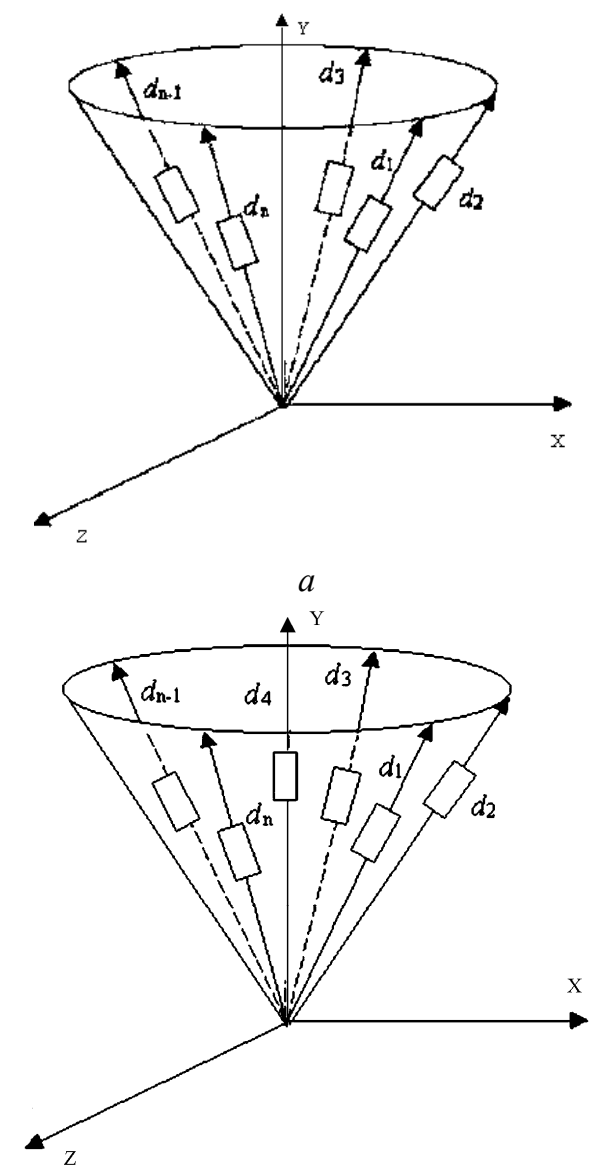

$b$

Fig. 2. Arrangement of redundant sensors along the cone's generators $(a)$ and along the cone's generators and axis of symmetry $(b)$

The main problem under such an approach to the redundancy of measuring reference frames is to determine the optimal orientation of the sensors. Consider some possible cases of building such orientation systems. We will build tables of directional cosines and analyze the accuracy of the obtained navigational information. We will denote the projections of kinematic parameters onto the basic reference frame as $a_{x}, a_{y}, a_{z}$. The projections of sensor measurements on the measuring reference frame are denoted $d_{1}, \ldots, d_{n}$. Directional cosines in the case of arranging four, five and six sensors along the cone's generators at an angle $\vartheta\left(54^{\circ} 44^{\prime}\right)$ to the axis of symmetry are presented in tab. 1 .
Table 1

Arrangement of sensors along the cone's generators at an angle $\vartheta$ to the axis of symmetry

\begin{tabular}{|l|l|c|c|}
\hline$d$ & $a_{x}$ & $a_{y}$ & $a_{z}$ \\
\hline \multicolumn{3}{|c|}{ Four sensors } \\
\hline$d_{1}$ & $\cos \pi / 4 \sin \vartheta$ & $\cos \vartheta$ & $-\cos \pi / 4 \sin \vartheta$ \\
\hline$d_{2}$ & $-\cos \pi / 4 \sin \vartheta$ & $\cos \vartheta$ & $-\cos \pi / 4 \sin \vartheta$ \\
\hline$d_{3}$ & $-\cos \pi / 4 \sin \vartheta$ & $\cos \vartheta$ & $\cos \pi / 4 \sin \vartheta$ \\
\hline$d_{4}$ & $\cos \pi / 4 \sin \vartheta$ & $\cos \vartheta$ & $\cos \pi / 4 \sin \vartheta$ \\
\hline \multicolumn{4}{|c|}{ Five $\operatorname{sensors}$} \\
\hline$d_{1}$ & 0 & $\cos \vartheta$ & $\sin \vartheta$ \\
\hline$d_{2}$ & $\sin 2 \pi / 5 \sin \vartheta$ & $\cos \vartheta$ & $\cos 2 \pi / 5 \sin \vartheta$ \\
\hline$d_{3}$ & $\sin / 5 \sin \vartheta$ & $\cos \vartheta$ & $-\cos \pi / 5 \sin \vartheta$ \\
\hline$d_{4}$ & $-\sin \pi / 5 \sin \vartheta$ & $\cos \vartheta$ & $-\cos \pi / 5 \sin \vartheta$ \\
\hline$d_{5}$ & $-\sin 2 \pi / 5 \sin \vartheta$ & $\cos \vartheta$ & $\cos 2 \pi / 5 \sin \vartheta$ \\
\hline \multicolumn{4}{|c|}{$\operatorname{six} \operatorname{sensors}$} \\
\hline$d_{1}$ & 0 & $\cos \vartheta$ & $\sin \vartheta$ \\
\hline$d_{2}$ & $\sin \pi / 3 \sin \vartheta$ & $\cos \vartheta$ & $\cos \pi / 3 \sin \vartheta$ \\
\hline$d_{3}$ & $\sin \pi / 3 \sin \vartheta$ & $\cos \vartheta$ & $-\cos \pi / 3 \sin \vartheta$ \\
\hline$d_{4}$ & 0 & $\cos \vartheta$ & $-\sin \vartheta$ \\
\hline$d_{5}$ & $-\sin \pi / 3 \sin \vartheta$ & $\cos \vartheta$ & $-\cos \pi / 3 \sin \vartheta$ \\
\hline$d_{6}$ & $-\sin \pi / 3 \sin \vartheta$ & $\cos \vartheta$ & $\cos \pi / 3 \sin \vartheta$ \\
\hline & 0 &
\end{tabular}

If three, four and five sensors are oriented along the cone's generators respectively and one sensor $\left(d_{4}, d_{5}, d_{6}\right.$ respectively) is oriented along the axis of symmetry, the directional cosines will correspond to tab. 2 .

Table 2

Arrangement of sensors along the cone's generators and along the axis of symmetry

\begin{tabular}{|l|c|c|c|}
\hline$d$ & $a_{x}$ & $a_{y}$ & $a_{z}$ \\
\hline $\begin{array}{c}d_{4}, \\
d_{5}, d_{6}\end{array}$ & 0 & 1 & 0 \\
\hline \multicolumn{4}{|c|}{ Three sensors } \\
\hline$d_{1}$ & 0 & $\cos \vartheta$ & $\sin \vartheta$ \\
\hline$d_{2}$ & $\cos \pi / 6 \sin \vartheta$ & $\cos \vartheta$ & $-\cos \pi / 3 \sin \vartheta$ \\
\hline$d_{3}$ & $-\cos \pi / 6 \sin \vartheta$ & $\cos \vartheta$ & $\cos \pi / 3 \sin \vartheta$ \\
\hline \multicolumn{4}{|c|}{ Four sensors } \\
\hline$d_{1}$ & $\cos \pi / 4 \sin \vartheta$ & $\cos \vartheta$ & $-\cos \pi / 4 \sin \vartheta$ \\
\hline$d_{2}$ & $-\cos \pi / 4 \sin \vartheta$ & $\cos \vartheta$ & $-\cos \pi / 4 \sin \vartheta$ \\
\hline$d_{3}$ & $-\cos \pi / 4 \sin \vartheta$ & $\cos \vartheta$ & $\cos \pi / 4 \sin \vartheta$ \\
\hline$d_{4}$ & $\cos \pi / 4 \sin \vartheta$ & $\cos \vartheta$ & $\cos \pi / 4 \sin \vartheta$ \\
\hline \multicolumn{4}{|c|}{ Five $\operatorname{sensors}$} \\
\hline$d_{1}$ & 0 & $\cos \vartheta$ & $\sin \vartheta$ \\
\hline$d_{2}$ & $\sin 2 \pi / 5 \sin \vartheta$ & $\cos \vartheta$ & $\cos 2 \pi / 5 \sin \vartheta$ \\
\hline$d_{3}$ & $\sin \pi / 5 \sin \vartheta$ & $\cos \vartheta$ & $-\cos \pi / 5 \sin \vartheta$ \\
\hline$d_{4}$ & $-\sin \pi / 5 \sin \vartheta$ & $\cos \vartheta$ & $-\cos \pi / 5 \sin \vartheta$ \\
\hline$d_{5}$ & $-\sin 2 \pi / 5 \sin \vartheta$ & $\cos \vartheta$ & $\cos 2 \pi / 5 \sin \vartheta$ \\
\hline
\end{tabular}


In the case of either a tetrahedron or a dodecahedron, taking into account that the angle between the sensors is $31^{\circ} 43^{\prime}$, we may write matrices of directional cosines in the form of tab. 3 .

The tab. 3 of directional cosines is determined on the basis of the following relationships:

$d_{1}=a_{x} \sin \vartheta+a_{y} \cos \vartheta$

$d_{2}=a_{y}$;

$d_{3}=-a_{x} \sin \pi / 6 \sin \vartheta+a_{y} \cos \vartheta-a_{z} \cos \pi / 6 \sin \vartheta ;$

$d_{4}=-a_{x} \sin \pi / 6 \sin \vartheta+a_{y} \cos \vartheta+a_{z} \cos \pi / 6 \sin \vartheta$

for the tetrahedron and

$d_{1}=a_{x} \cos \gamma-a_{y} \sin \gamma$

$d_{2}=a_{x} \cos \gamma+a_{y} \sin \gamma$;

$d_{3}=a_{y} \cos \gamma-a_{z} \sin \gamma$;

$d_{4}=a_{y} \cos \gamma+a_{z} \sin \gamma$

$d_{5}=a_{z} \cos \gamma-a_{x} \sin \gamma$

$d_{6}=a_{z} \cos \gamma+a_{x} \sin \gamma$

for the dodecahedron.

Table 7

Arrangement of sensors relative to the faces of a tetrahedron and a dodecahedron

\begin{tabular}{|c|c|c|c|}
\hline$d$ & $a_{x}$ & $a_{y}$ & $a_{z}$ \\
\hline \multicolumn{3}{|c|}{ Tetrahedron } \\
\hline$d_{1}$ & $\sin \vartheta$ & $\cos \vartheta$ & 0 \\
\hline$d_{2}$ & 0 & 1 & 0 \\
\hline$d_{3}$ & $-\sin \pi / 6 \sin \vartheta$ & $\cos \vartheta$ & $-\cos \pi / 6 \sin \vartheta$ \\
\hline$d_{4}$ & $-\sin \pi / 6 \sin \vartheta$ & $\cos \vartheta$ & $\cos \pi / 6 \sin \vartheta$ \\
\hline \multicolumn{4}{|c|}{ Dodecahedron } \\
\hline$d_{1}$ & $\cos \gamma$ & $-\sin \gamma$ & 0 \\
\hline$d_{2}$ & $\cos \gamma$ & $\sin \gamma$ & 0 \\
\hline$d_{3}$ & 0 & $\cos \gamma$ & $-\sin \gamma$ \\
\hline$d_{4}$ & 0 & $\cos \gamma$ & $\sin \gamma$ \\
\hline$d_{5}$ & $-\sin \gamma$ & 0 & $\cos \gamma$ \\
\hline$d_{6}$ & $\sin \gamma$ & 0 & $\cos \gamma$ \\
\hline
\end{tabular}

О.А. Сущенко, О.В.Плотніков

Визначення орієнтації рухомого об'єкта за допомогою надмірних неортогональних систем датчиків

Розглянуто особливості визначення орієнтації рухомого об'єкта за допомогою надмірних неортогональних систем датчиків. Показано переваги цього способу підвищення надійності навігаційних систем. Розглянуто різні способи орієнтації неортогональних систем і виконано їхній порівняльний аналіз.

О.А. Сущенко, А.В.Плотников

Определение ориентации подвижного объекта с помощью избыточных неортогональных систем датчиков Рассмотрены особенности определения ориентации подвижного объекта посредством избыточных неортогональных систем датчиков. Показаны преимущества этого способа повышения надежности навигационных систем. Проанализированы разные способы ориентации избыточных неортогональных систем и выполнен их сравнительный анализ.
The results of a comparative analysis of different non-orthogonal redundant systems of sensors are presented in tab. 4. Here, the trace of matrix $\mathbf{H}=\left(\mathbf{L}^{\mathrm{T}} \mathbf{L}\right)^{-1}$ is considered as one of the characteristics of a redundant system [5], where $\mathbf{L}$ is a matrix of directional cosines.

Table 9

\section{Different non-orthogonal redundant systems of sensors}

\begin{tabular}{|c|c|c|}
\hline $\begin{array}{c}\text { Number of } \\
\text { sensors }\end{array}$ & $\begin{array}{c}\text { Trace of matrix } \\
\mathbf{H}\end{array}$ & $\begin{array}{c}\text { Redundancy on } \\
\text { axes } x, y, z\end{array}$ \\
\hline 4 & 2,25 & $3,3,3$ \\
\hline 5 & 1,8 & $3,4,4$ \\
\hline 6 & 1,78 & $3,5,5$ \\
\hline 4 & 4,0 & $1,3,2$ \\
\hline 5 & 1,92 & $3,4,3$ \\
\hline 6 & 1,57 & $3,5,4$ \\
\hline 4 & 2,5 & $2,3,1$ \\
\hline 6 & 1,5 & $3,3,3$ \\
\hline
\end{tabular}

\section{Conclusion}

Orientation of different non-orthogonal redundant determined.

A comparative analysis of different ways of building redundant systems of sensors is carried out.

1. Андреев В.Д. Теория инерциальной навигации. Автоматические системы. - М.: Наука, 1967. - 416 с. 2. Несенюк Л.П. Степанов О.А. Состояние и теория развития интегрированных систем ориентации и навигации // Гиротехнологии, навигация, управление движением и конструирование авиационно-космической техники: Сб. докл. - К.: "КПІ", 2003 - Ч. 1 - С. 16-32.

М.: Машиностроение, 1975. - 180 c.

4. Епифанов А.Д. Избыточные системы управления летательными аппаратами. - М.: Машиностроение, 1978. - 144 с.

5. Кошляков В.Н. Задачи динамики твердого тела и прикладной теории гироскопов. - М.: Наука, 1985. -288 c.

The editors received the article on 9 March 2005. configurations of kinematic parameter sensors is 